\title{
The Effect of Locally Administered Fibrinolytic Drugs Following Aneurysmal Subarachnoid Hemorrhage : A Meta-Analysis with Eight Randomized Controlled Studies
}

\author{
Kyoung Min Jang,, Hyun Ho Choi, ${ }^{1,2}$ Taek Kyun Nam, ${ }^{1}$ Yong Sook Park, Jeong Taik Kwon, Jun Soo Byun, ${ }^{3}$ Doyeon Hwang ${ }^{4}$ \\ Department of Neurosurgery, Chung-Ang University Hospital, Chung-Ang University College of Medicine, Seoul, Korea \\ Department of Neurosurgery, Kangwon National University School of Medicine, Chuncheon, Korea \\ Department of Radiology, ${ }^{3}$ Chung-Ang University Hospital, Chung-Ang University College of Medicine, Seoul, Korea \\ Department of Internal Medicine and Cardiovascular Center, ${ }^{4}$ Seoul National University Hospital, Seoul, Korea
}

Objective : Rapid dissolution of blood clots reduces vasospasm and hydrocephalus after subarachnoid hemorrhage (SAH), and locally administered fibrinolytic drugs (LAFDs) could facilitate the dissolution. However, the efficacy of LAFDs remains controversial. The aim of this meta-analysis was to determine the efficacy of LAFDs for vasospasm and hydrocephalus and in clinical outcomes.

Methods : From PubMed, EMBASE, and Cochrane database, data were extracted by two authors. Meta-analysis was performed using a random effect model. Inclusion criteria were patients who had LAFDs with urokinase-type or recombinant tissue-plasminogen activator after SAH in comparison with medically untreated patients with fibrinolytic drugs. We only included randomized controlled trials (RCTs) in this analysis. The outcomes of interest were vasospasm, hydrocephalus, mortality, and 90 -day unfavorable functional outcome.

Results : Data from eight RCTs with 550 patients were included. Pooled-analysis revealed that the LAFDs were significantly associated with lower rates of vasospasm (LAFDs group vs. control group, $26.5 \%$ vs. 39.2\%; odds ratio [OR], 0.48 ; $95 \%$ confidence interval [CI], $0.32-$ 0.73); hydrocephalus (LAFDs group vs. control group, $26.0 \%$ vs. $31.6 \% ; \mathrm{OR}, 0.54 ; 95 \% \mathrm{Cl}, 0.32-0.91$ ); and mortality (LAFDs group vs. control group, $10.5 \%$ vs. $15.7 \%$; $\mathrm{OR}, 0.58 ; 95 \% \mathrm{Cl}, 0.34-0.99$ ). The proportion of 90 -day unfavorable outcomes was lower in the LAFDs group (LAFDs group vs. control group, $32.7 \%$ vs. $43.5 \%$; $\mathrm{OR}, 0.55 ; 95 \% \mathrm{Cl}, 0.37-0.80)$.

Conclusion : This meta-analysis with eight RCTs indicated that LAFDs were significantly associated with lower rates of vasospasm and hydrocephalus after SAH. Thus, LAFDs could consequently reduce mortality and improve clinical outcome after SAH.

Key Words : Subarachnoid hemorrhage · Fibrinolytic agents · Plasminogen activators · Vasospasm, Intracranial · Hydrocephalus.

\section{INTRODUCTION}

Aneurysmal subarachnoid hemorrhage (SAH) can cause disability and has a $30-40 \%$ mortality rate ${ }^{3)}$. Secondary com- plications including cerebral vasospasm and hydrocephalus are documented as significant risk factors of poor clinical outcomes after $\mathrm{SAH}^{4)}$. Thus, vasospasm and hydrocephalus are major concerns regarding prevention of secondary morbidity

- Received : June 3, 2020 - Accepted : July 29, 2020

- Address for reprints : Hyun Ho Choi

Department of Neurosurgery, Chung-Ang University Hospital, Chung-Ang University College of Medicine, 102 Heukseok-ro, Dongjak-gu, Seoul 06973, Korea Tel : +82-2-6299-1608, Fax : +82-2-6299-2017, E-mail : ayohyunho@naver.com, ORCID : https://orcid.org/0000-0003-1170-6829 
and mortality.

The clot volume of the intraventricular and intracisternal hemorrhage after aneurysm rupture has been suggested as an independent risk factor for predicting the occurrence of vasospasm and hydrocephalus ${ }^{21)}$. Moreover, rapid clot clearance rate is considered to play an important role in reducing the incidence of vasospasm and hydrocephalus. Plasminogen activator induces the formation of fibrin degradation products by converting plasminogen to plasmin and contributes to the thrombolytic process ${ }^{22)}$. Representative fibrinolytic drugs such as recombinant tissue-plasminogen activator (rt-PA) and urokinase-type plasminogen activator (UK) can be administered locally to accelerate clot clearance via extraventricular drainage (EVD) or intrathecal catheter. Although many studies have assessed the preventive efficacy of locally administered fibrinolytic drugs (LAFDs) for vasospasm and hydrocephalus, the results are not yet conclusive $e^{3,6,7,9,14,17,19,31)}$. Previously published meta-analyses for LAFDs also show limited evidence because of inclusion of observational studies ${ }^{1,26)}$. Therefore, the aims of this study were to determine the effectiveness of LAFDs by performing a meta-analysis that only included randomized controlled trials (RCTs).

\section{MATERIALS AND METHODS}

A study of this type, such as meta-analysis, does not require informed consent or Institutional Review Board's approval. The authors applied a protocol designed prior to the study that was in accordance with the Preferred Reporting Items for Systematic Reviews and Meta-analysis (PRISMA) guidelines (Supplementary Table 1).

\section{Data sources and searches}

Two independent investigators (coauthors on this study) searched PubMed, EMBASE, the Cochrane Central Register of Controlled Trials, and relevant web sites using phased and concrete search terms (Supplementary Method 1). The electronic search strategy was complemented by a manual screening of references cited in the included articles such as recent reviews, editorials, and meta-analyses. There were no restrictions on the language of publication, study period, or sample size.

\section{Eligibility criteria and study selection}

The goals of the search were to identify articles that met the following inclusion criteria : 1) prospective study design, namely RCTs (with or without blinding); 2) participants with ruptured aneurysmal SAH and treated with endovascular coiling or conventional clipping; 3) trials with administration of LAFDs, including rt-PA or UK, after aneurysmal repair; 4) injection administrations via intraventricular, intracisternal, intrathecal routes; and 5) a medically untreated group with fibrinolytic drugs as the control. We excluded studies based on the following criteria : 1) those with a retrospective study design including observational, case-control, cohort study, case series, or reports; 2) those with participants having non-aneurysmal SAH or intracerebral hemorrhage; 3) trials involving patients with local administration of a drug other than rt-PA or UK; 4) those with intravenous route of administration; and 5) those with control groups comprising subjects with local administration of another drug other than rt-PA or UK. Two investigators independently screened the titles and abstracts, identified duplicates, reviewed full articles, and determined their eligibility. In the event of discrepancy between the reviewers, consensus was established through discussion with the corresponding author.

\section{Data extraction and quality assessment}

Data were obtained independently in duplicate by two investigators. A standardized form was utilized to extract study characteristics, study design, sample size, age, sex, and severity index that was (calculated based on the World Federation of Neurosurgical Societies score). In the case of studies using other grading systems such as the Glasgow coma scale, and Hunt-Hess grade, the investigators converted the data to comply with the World Federation of Neurosurgical Societies score considered to be most similar" ${ }^{5}$. In addition, the authors recorded the Fisher's grade, the surgical procedure of aneurysmal repair involving clipping or coiling, and any intervention protocol including type, dose, route, and duration of the administered drugs.

The authors established the outcomes including the incidence of vasospasm and hydrocephalus, all-cause mortality during hospital day (mortality), and poor clinical outcome at postictal 90 days (unfavorable outcome). In this study, we defined vasospasm as narrowing of any large vessel diameter by $>30 \%$ from the baseline, on digital subtraction angiography 
and computed tomography ${ }^{7}$, and hydrocephalus as a permanent shunt insertion in a patient with symptoms of neurologic deteriorations including alteration of consciousness, motor deficit, and gait disturbance ${ }^{26}$. The unfavorable outcome was defined as Glasgow outcome scale of 1-3 and/or modified Rankin score of 3-62).

The quality of eligible studies was assessed using the Cochrane Collaboration's tool for assessing the risk of bias (RoB) for RCTs using the Review Manager (RevMan version 5.3 software; The Cochrane Collaboration, Oxford, UK). The two authors assessed the study quality through RoB criteria including selection, performance, detection, attrition, reporting, and other biases.

\section{Data synthesis}

Statistical analysis was performed using Review Manager and STATA/SE 15.0 (Stata Corp LP, College Station, TX, USA). The outcomes including vasospasm, hydrocephalus, mortality, and unfavorable outcome at 90 days were analyzed using the random effect model with generic inverse variance method, and were presented with the odds ratio (OR) and 95\% confidence interval (CI) as a summary statistic. We excluded data presented as medians with interquartile range and those that did not report the standard deviation values. Subgroup analyses were conducted to explore the confounder of a different type or route of administered drugs. The type consisted of rt-PA and UK, while the route was divided into EVD or intrathecal administration.

Statistical heterogeneity was estimated using the $\mathrm{I}^{2}$ statistic, which measures the degree of inconsistency across studies in a meta-analysis. $\mathrm{I}^{2}<25 \%,<50 \%$, and $>75 \%$ were considered to indicate low, moderate, and severe heterogeneity, respectively. The authors investigated publication bias by funnel plot asymmetry and Egger's and Begg's tests. When visual asymmetry of the funnel plot was suspected, the trim-and-fill method was used to estimate an adjusted OR. Two-sided $p$-values $<0.05$ were considered to indicate statistical significance.

\section{RESULTS}

\section{Search results}

From the initial 636 candidate articles, 355 duplicated articles were excluded. Among the 281 remaining articles, 34 arti- cles were extracted for full article review. Finally, eight articles that enrolled 550 patients met the inclusion criteria (Fig. 1). The context of excluded articles is summarized in the Supplementary Method 2. All eight articles reported vasospasm, mortality, and unfavorable outcome, while hydrocephalus was only documented in six. Intervention was carried out by rt-PA administration in six and UK in two articles. In respect of administration route, four studies performed the injection through the EVD, and the other four adopted intrathecal route. The major characteristics of the eight individual articles are summarized in Table 1.

\section{RoB of included studies}

The RoB assessment of RCTs is presented in an organized table (Fig. 2). Among the eight RCTs, two clearly specified the method of random sequence generation and allocation concealment, including computerized software and use of sealed envelope. While the remaining six RCTs conducted randomization, they lacked a complete and adequate statement regarding concealment. Although these RCTs represented an unclear RoB, the endpoints of this study, objective findings, and the measurement of outcomes were unlikely to be influenced by the blinding. Only one study with a high RoB with respect to attrition and reporting bias was reported. Other than these two items, no high RoB was observed in any of the other studies. Supplementary Table 2 presents concrete support for judgment of RoB criteria.

\section{Comparison of vasospasm and hydrocephalus}

The conducted rates of vasospasm incidence in the pooled analysis were $26.5 \%$ in LAFDs group and 39.2\% in control group. Integrating the results of all eight RCTs showed that the LAFDs was significantly associated with a reduced risk of vasospasm without obvious heterogeneity (pooled OR, 0.48; 95\% CI, 0.32-0.73; $p=0.366 ; \mathrm{I}^{2}=0.0 \%$ ) (Fig. 3A). Distinct publication bias was not identified, supported by visual symmetry of the funnel plot and the Egger's and Begg's tests (Supplementary Fig. 1A).

The rates of incidence for hydrocephalus in the pooled analysis was $26.0 \%$ in LAFDs group and $31.6 \%$ in control group. Combined results of all eight RCTs showed that the LAFDs were significantly associated with lower rates of hydrocephalus (pooled OR, 0.54; 95\% CI, 0.32-0.91; $p=0.441$; $\mathrm{I}^{2}=0.0 \%$ ) (Fig. $3 \mathrm{~B}$ ). The authors detected visual asymmetry in 


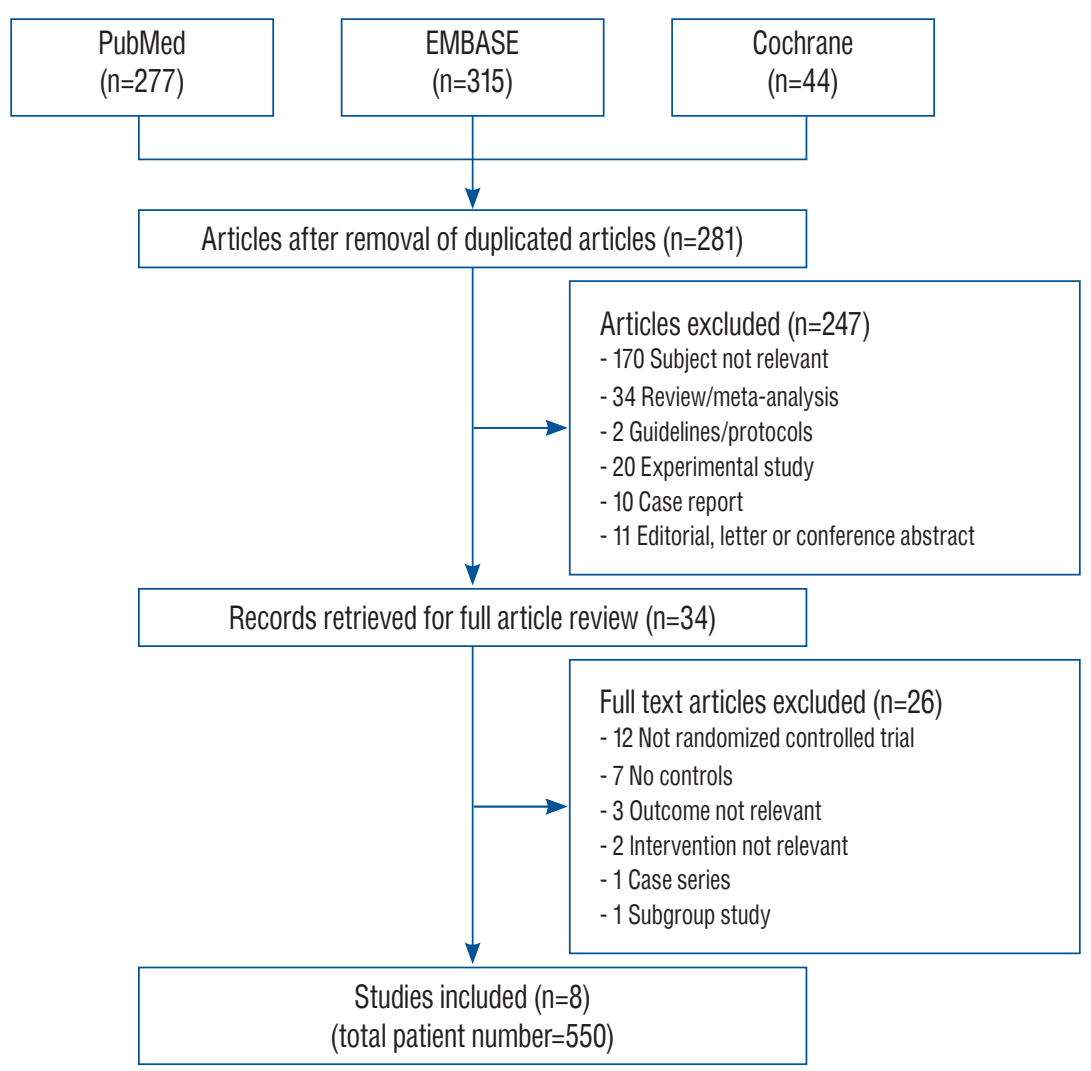

Fig. 1. Flow diagram for the selection of relevant studies.

the funnel plot and applied the trim-and-fill correction (Supplementary Fig. 1B). In comparison to the pooled OR, the adjusted OR showed no obvious heterogeneity (adjusted OR, $0.51 ; p=0.392$ ).

\section{Comparison of mortality and unfavorable outcome}

The observed rates of mortality in pooled analysis were $10.5 \%$ in LAFDs group and $15.7 \%$ in control group. A pairwise meta-analysis with eight RCTs showed that LAFDs were significantly related with lower rates in mortality without statistical heterogeneity (pooled OR, 0.58; 95\% CI, 0.34-0.99; $p=0.992 ; I^{2}=0.0 \%$ ) (Fig. 3C). Publication bias was not identified regarding the symmetrical funnel plot supported by the Egger's and Begg's tests (Supplementary Fig. 1C).

The analysis revealed that the individual rate of unfavorable outcome was $32.7 \%$ in LAFDs group and $43.5 \%$ in control group. A pairwise analysis of all eight RCTs showed that LAFDs were significantly associated with the higher rates in unfavorable outcome with low heterogeneity (pooled OR, 0.55; 95\% CI, 0.37-0.80; $p=0.134 ; \mathrm{I}^{2}=37.0 \%$ ) (Fig. 3D). Although visual asymmetry was observed in funnel plot, the ad- justed OR showed no significant differences as compared with pooled OR owing to the trim-and-fill correction (adjusted OR, $0.47 ; p=0.330$ ) (Supplementary Fig. 1D).

\section{Subgroup analysis}

Subgroup analysis was performed by the type and route of administered drugs (Fig. 4). There were no statistical differences regarding the type of drugs (rt-PA vs. UK) prescribed for the vasospasm (pooled RR, 0.26 vs. 0.63 ; $p=0.366$ ); hydrocephalus (pooled RR, 0.31 vs. $0.81 ; p=0.441$ ); mortality (pooled RR, 0.71 vs. $0.53 ; p=0.180$ ): and unfavorable outcome (pooled RR, 0.81 vs. $0.31 ; p=0.134$ ). In addition, the route of administration not affect all outcomes. All subgroup analyses showed that there was no significant heterogeneity.

\section{DISCUSSION}

Vasospasm on angiography is typically detected in about $66 \%$ of patients with aneurysmal SAH within 3-14 days following initial rupture ${ }^{3)}$. Blood clots in the intraventricular and 
The Fibrinolytic Drugs Following SAH | Jang KM, et al.
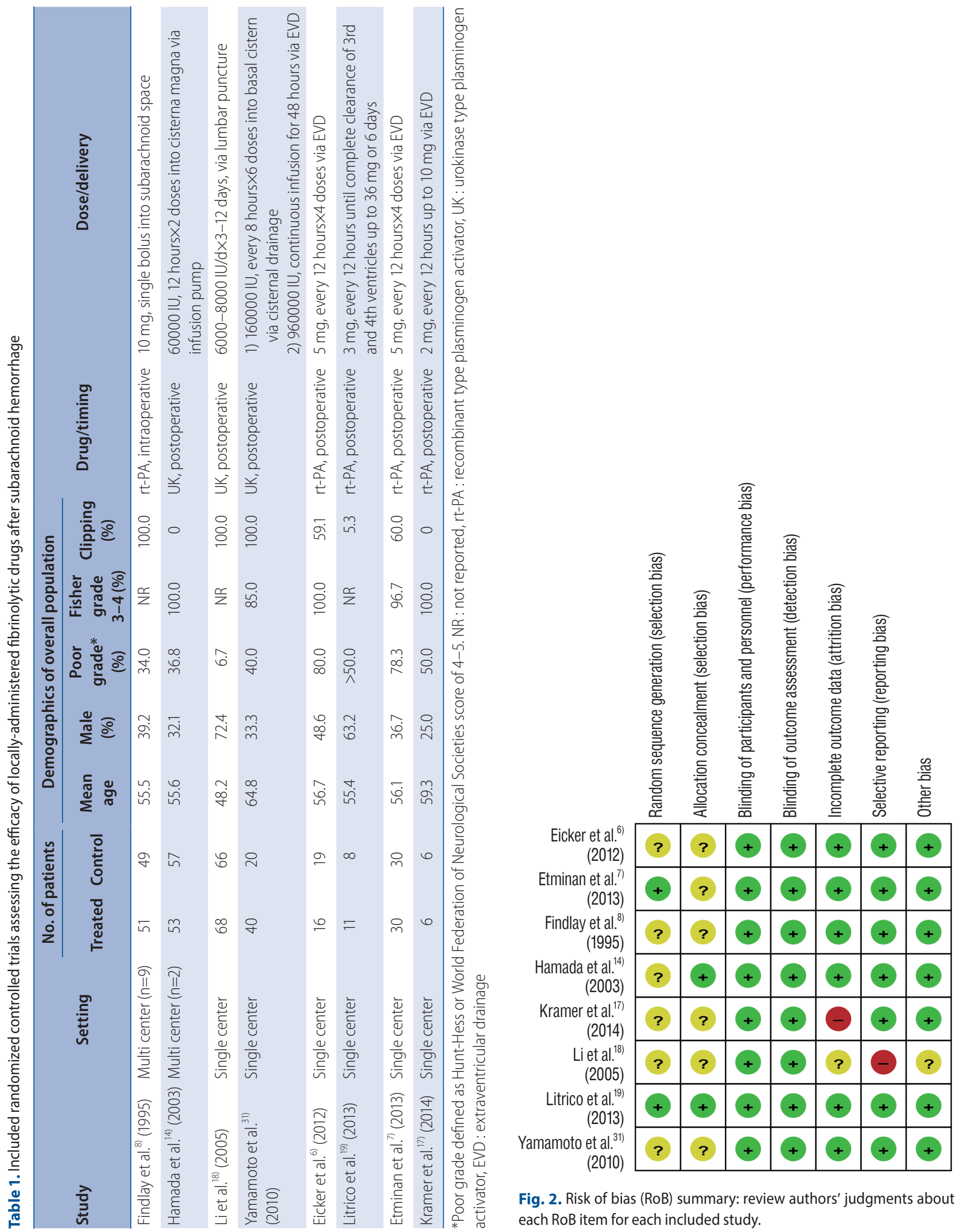

Fig. 2. Risk of bias (RoB) summary: review authors' judgments about each RoB item for each included study. 


\begin{tabular}{l} 
Study \\
\hline Findlay et al. ${ }^{8)}(1995)$ \\
Hamada et al. ${ }^{14)}(2003)$ \\
Li et al..$^{(8)}(2005)$ \\
Yamamoto et al..$^{31)}(2010)$ \\
Eicker et al. ${ }^{6)}(2012)$ \\
Litrico et al..$^{(9)}(2013)$ \\
Etminan et al..$^{7}(2013)$ \\
Kramer et al. ${ }^{17)}(2014)$ \\
Heterogeneity $\left(\left(^{2}=0.0 \%, p=0.366\right)\right.$
\end{tabular}

(A)

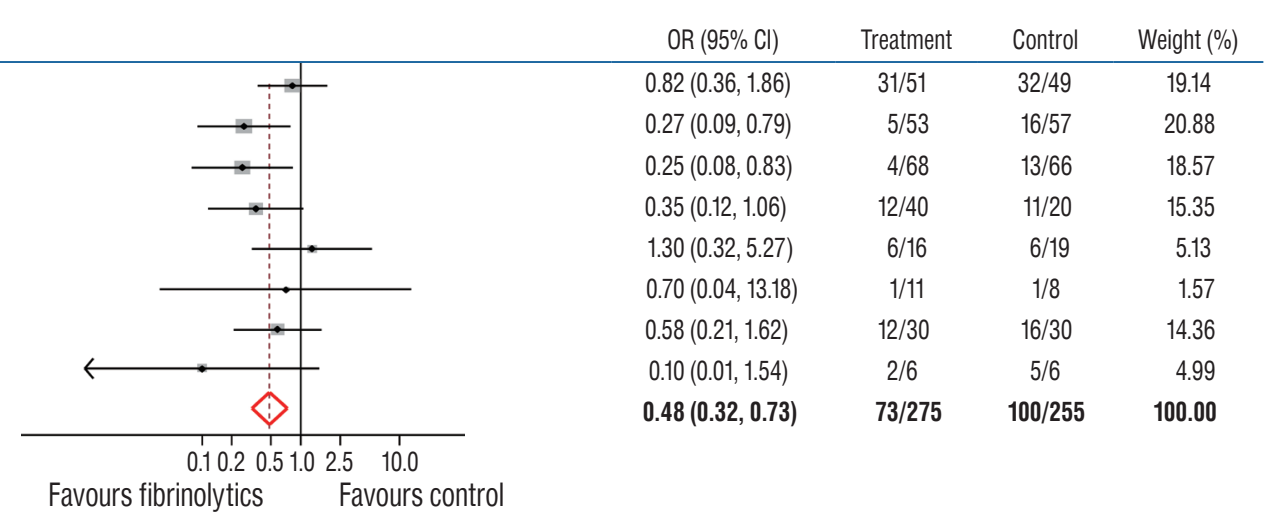

Study

Hamada et al. ${ }^{14)}$ (2003)

Li et al. ${ }^{18)}(2005)$

Yamamoto et al. ${ }^{31)}(2010)$

Litrico et a. ${ }^{19)}(2013)$

Etminan et al. ${ }^{7)}(2013)$

Kramer et al. ${ }^{17)}(2014)$

Heterogeneity $\left(I^{2}=0.0 \%, p=0.441\right)$

(B)

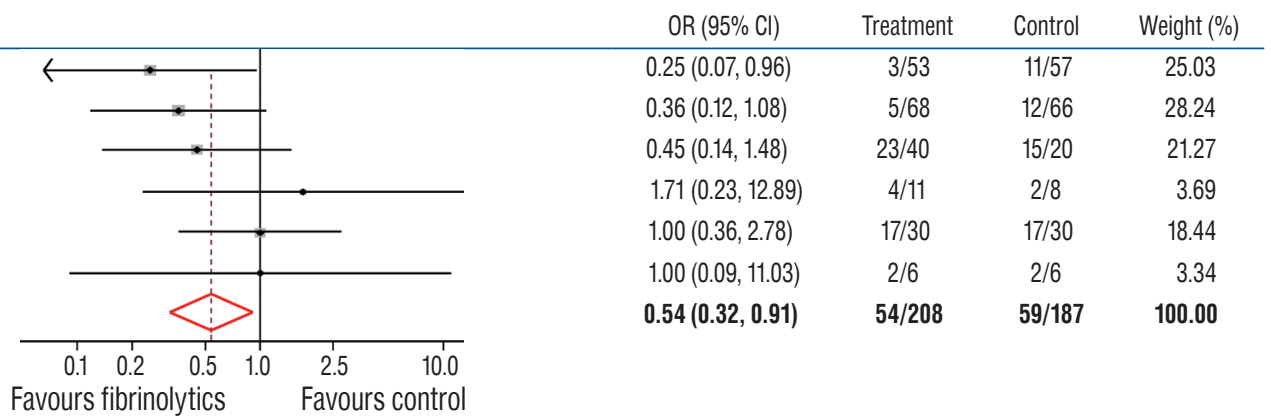

Study

Findlay et al..$^{8)}(1995)$

Hamada et al. ${ }^{14)}$ (2003)

Li et al. ${ }^{18)}$ (2005)

Yamamoto et al..$^{31)}$ (2010)

Eicker et al. ${ }^{6)}$ (2012)

Litrico et al. ${ }^{19)}(2013)$

Etminan et al. ${ }^{7)}$ (2013)

Kramer et al. ${ }^{17)}(2014)$

Heterogeneity $\left(l^{2}=0.0 \%, p=0.992\right)$

(C)

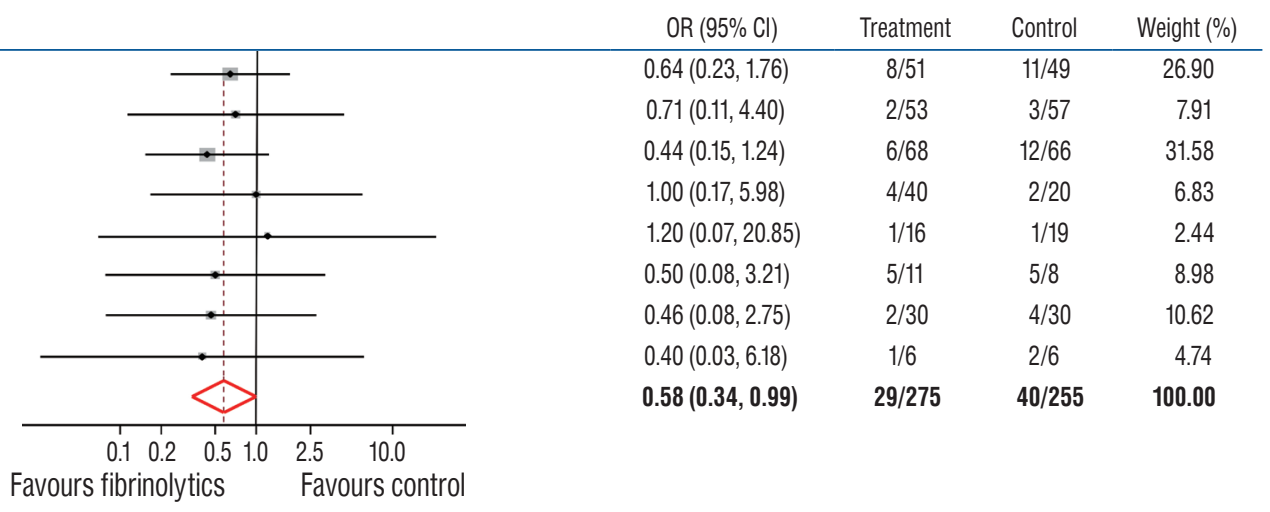

\begin{tabular}{l}
\hline Findlay et al. ${ }^{8}(1995)$ \\
Hamada et al..$^{(4)}(2003)$ \\
Li et al..$^{(8)}(2005)$ \\
Yamamoto et al..$^{31)}(2010)$ \\
Eicker et al. ${ }^{6)}(2012)$ \\
Litrico et al..$^{(9)}(2013)$ \\
Etminan et al..$^{7)}(2013)$ \\
Kramer et al. ${ }^{17)}(2014)$ \\
Heterogeneity $\left(\left(^{2}=37.0 \%, p=0.134\right)\right.$
\end{tabular}

(D)

\begin{tabular}{|c|c|c|c|c|c|}
\hline & & OR $(95 \% \mathrm{Cl})$ & Treatment & Control & Weight (\%) \\
\hline & & $1.05(0.43,2.53)$ & $14 / 51$ & $13 / 49$ & 13.29 \\
\hline & & $0.32(0.11,0.96)$ & $5 / 53$ & $14 / 57$ & 16.88 \\
\hline & & $0.30(0.14,0.64)$ & $15 / 68$ & $32 / 66$ & 34.97 \\
\hline & & $0.60(0.20,1.79)$ & $19 / 40$ & $12 / 20$ & 11.60 \\
\hline & & $0.21(0.05,0.90)$ & $6 / 16$ & $14 / 19$ & 11.05 \\
\hline$\leftarrow$ & & $0.64(0.05,8.62)$ & $9 / 11$ & $7 / 8$ & 2.04 \\
\hline & & $1.51(0.54,4.24)$ & $19 / 30$ & $16 / 30$ & 8.10 \\
\hline & & $1.00(0.10,9.61)$ & $3 / 6$ & $3 / 6$ & 2.07 \\
\hline & & $0.55(0.37,0.80)$ & $90 / 275$ & $111 / 255$ & 100.00 \\
\hline $\begin{array}{cccc}0.1 & 0.2 & 0.5 & 1.0 \\
\text { avours fibrinolytics } & \end{array}$ & $\begin{array}{ll}2.5 & 10.0 \\
\text { Favours control }\end{array}$ & & & & \\
\hline
\end{tabular}

Fig. 3. Efficacy of locally administered fibrinolytic drugs in the prevention of outcomes. Tests for heterogeneity are described using $\mathrm{I}^{2}$ and $p$ value. A : Vasospasm. B:Hydrocephalus. C: Mortality. D: Unfavorable clinical outcome at 90 days. OR : odds ratio, $\mathrm{Cl}$ : confidence interval. 
intracisternal areas after hemorrhage induces vasoconstriction and results in angiographic narrowing. Released oxyhemoglobin and blood-breaking products derived from the clot induce the oxygen-free reactions, and cause inflammation and endothelial injury, which in turn lead to endothelin-1 and nitric oxide expression. These cytokines result in vasospasms by way of microthrombi, vascular constriction, and extracellular matrix inflammation. Thus, thickness, density, location, and duration of the clot were indicated as risk factors for vaso$\operatorname{spasm}^{20)}$. Clot volume and clearance rate were also reported as independent predictors of vasospasm in previous studies. Reilly et al. ${ }^{25)}$ indicated the initial clot volume and declined rate as significant predictors of vasospasm. Hänggi et al. ${ }^{13}$ also reported that accelerated clot clearance with intrathecal lavage reduced the incidence of vasospasms. Plasminogen activator was presumed to facilitate the thrombolytic process, which induces clot-lysis ${ }^{22}$. Accordingly, LAFDs, including rt-PA or UK via EVD or intrathecal administration has been proposed as a postoperative treatment modality to prevent vasospasm accelerating the clot clearance rate ${ }^{8,9)}$. Various studies have been conducted to investigate the efficacy of LAFDs. Eicker et al. ${ }^{6}$ studied 35 patients who underwent surgery with aneurysmal SAH. Among them, 16 patients received four, 12-hourly 5-mg rt-PA doses via EVD. The clot clearance rate was signifi- cantly higher in patients with rt-PA treatment than in those ( $48.3 \%$ vs. $21.3 \%, p=0.003)$. Kawamoto et al. ${ }^{16)}$ reported the efficacy of cisternal irrigation with UK. Hamada et al. ${ }^{14)}$ designed an RCT including 110 patients who underwent cisternal irrigation with UK and showed a significantly low incidence of vasospasm. In the current study, we performed pooled analysis to compare the incidence of vasospasm between in the LAFD group and control group. Our results showed that patients treated with LAFDs were significantly associated with the lower incidence rates of vasospasm (LAFDs group vs. control group, 26.5\% vs. 39.2\%; OR, 0.48; 95\% CI, 0.32-0.73). Therefore, we believe that LAFDs seems likely to play an important role in reducing the risk of vasospasm through the acceleration of clot clearance.

The incidence of hydrocephalus after SAH ranges from 15\% to $20 \%$ and is considered a major source of morbidity and mortality following $\mathrm{SAH}^{10)}$. Ventricular involvement occurs in $30 \%$ of SAH cases ${ }^{15)}$, and intraventricular hemorrhage (IVH) is known as an independent predictor of hydrocephalus after $\mathrm{SAH}^{27,30)}$. From the pathophysiologic perspective, the occurrence of IVH-induced hydrocephalus is understood to develop via two mechanisms-an obstructive hydrocephalus from IVH and blood clot by inhibiting the outflow of cerebrospinal fluid (CSF) following SAH, and a compromised reabsorption of

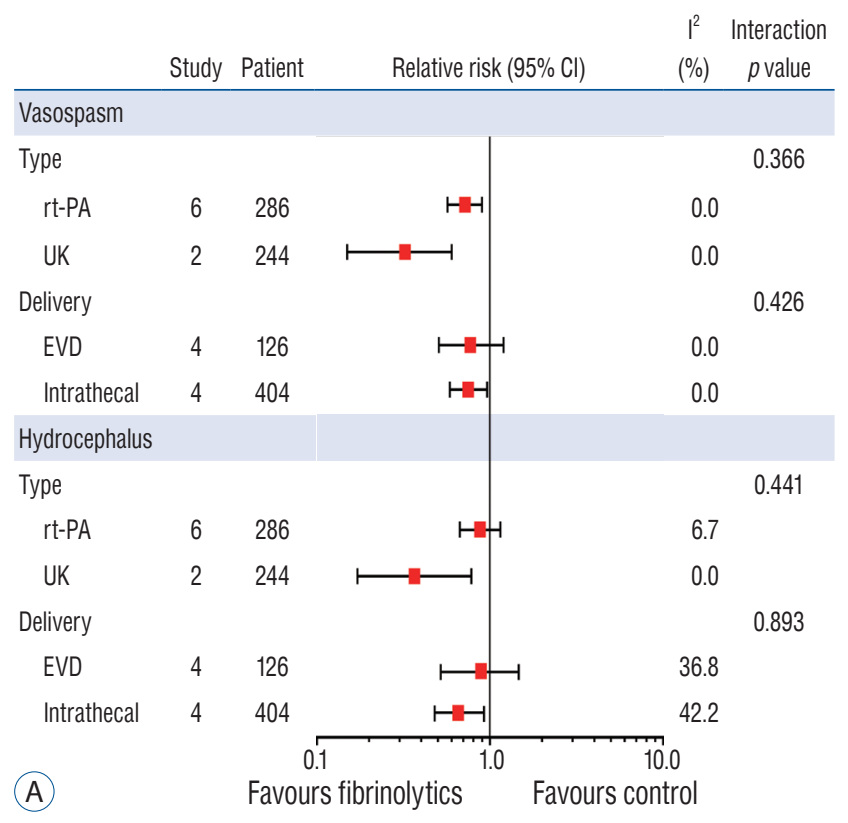

\begin{tabular}{|c|c|c|c|c|c|}
\hline & Study & Patient & Relative risk (95\% Cl) & $\begin{array}{l}1^{2} \\
(\%)\end{array}$ & $\begin{array}{c}\text { Interaction } \\
p \text { value }\end{array}$ \\
\hline \multicolumn{6}{|l|}{ Mortality } \\
\hline Type & & & & & 0.180 \\
\hline rt-PA & 6 & 286 & $\longmapsto+$ & 0.0 & \\
\hline UK & 2 & 244 & $\longmapsto-1$ & 0.0 & \\
\hline Delivery & & & & & 0.814 \\
\hline EVD & 4 & 126 & $\longmapsto-1$ & 0.0 & \\
\hline Intrathecal & 4 & 404 & $\longmapsto-1$ & 0.0 & \\
\hline \multicolumn{6}{|c|}{ Unfavorable outcome } \\
\hline Type & & & & & 0.134 \\
\hline rt-PA & 6 & 286 & $\longmapsto-1$ & 6.7 & \\
\hline UK & 2 & 244 & $\longmapsto$ & 0.0 & \\
\hline Delivery & & & & & 0.159 \\
\hline EVD & 4 & 126 & $\longmapsto$ & 36.8 & \\
\hline Intrathecal & 4 & 404 & $\longmapsto$ & 42.2 & \\
\hline (B) & & Favour & ibrinolytics 1.0 Favou & & \\
\hline
\end{tabular}

Fig. 4. Subgroup analysis : comparison of the efficacy of locally administered fibrinolytic drugs with respect to type and route of delivery. Interaction $p<0.05$ indicates significant difference between the two groups. A : Vasospasm, hydrocephalus. B : Mortality, unfavorable outcome. $\mathrm{Cl}$ : confidence interval, rt-PA : recombinant-type plasminogen activator, UK: urokinase-type plasminogen activator, EVD: extraventricular drainage. 
CSF with subarachnoid granulations. Acute hydrocephalus is enhanced by direct blockade of the CSF pathways by bloodbreaking products derived from an intraventricular clot; this non-communicating hydrocephalus usually presents in an acute setting. The latter is generally observed in the chronic setting by inflammation-mediated adhesions that inhibit the reuptake of CSF. Wilson et al. ${ }^{30)}$ reported that the presence of IVH was strongly associated with hydrocephalus and unfavorable outcome. The authors suggested that a combination of acute obstruction of CSF and chronic inflammation of granulations or choroid plexus results in hydrocephalus following SAH. Therefore, rapid removal of IVH and its products is considered to play an important role in preventing hydrocephalus; accordingly, the efficacy of LAFDs in clot-lysis for treating hydrocephalus after SAH has been investigated in previous studies $^{14,17-19,29)}$. Given these findings, rapid elimination of IVH following SAH have been presumed to reduce the occurrence of hydrocephalus, and previous studies have reported a reduction in hydrocephalus risk with intraventricular rt-PA administration $^{24,28)}$. Our results were in line with the previous studies. Pooled-analysis with eight RCTs also presented that clot lysis using LAFDs identifies a $46 \%$ decrease in the absolute risk of hydrocephalus occurrence compared to the control (OR, 0.54; 95\% CI, 0.32-0.91).

Vasospasm and hydrocephalus are strongly associated with increased rates in mortality and unfavorable outcome ${ }^{4,20)}$. In a systematic review of literature, Dorsch ${ }^{4)}$ noted that patients with vasospasm showed a significantly high risk of mortality as comparison to those without. Hydrocephalus is also considered an independent risk factor for other unfavorable outcomes and death due to ventricular enlargement and increased intracranial pressure $^{30)}$. Giraldo et al. ${ }^{12)}$ reported that the presence of hydrocephalus was a significant risk factor. In the systematic review by Karamanakos et al. ${ }^{15)}$, hydrocephalus was determined as an independent risk factor for delayed mortality following SAH. In the present meta-analysis, significant association with lower rates of mortality (OR, 0.58; 95\% CI, 0.34-0.99) and unfavorable outcome (OR, 0.55; 95\% CI, $0.37-0.80)$ were indicated. In conclusion, LAFDs can be used to lowering the incidence rates of vasospasm or hydrocephalus, and would be consequently reduce the mortality rates and unfavorable outcome.

Our study has some limitations. First, this analysis was conducted with the inconsistent protocol of the included studies (e.g., consistent regimen for dose, duration, and timing of initiation for each study). Additionally, the different included populations (e.g., the proportion of low or high Hunt-Hess grade) could not be limited. Although the included trials documented randomized or controlled characteristics of design, the differences in treatment protocols and populations between individual groups may have confounded estimates of the efficacy on postoperative fibrinolytic drugs. Second, it is possible that unmeasured factors such as the operator's skill may have acted as a confounder, which could affect the outcome. The safety of LAFDs were still debated due to the potential of inducing adverse effects such as hemorrhage or neurotoxicity $^{23)}$. In contrast, there was a report that LAFDs, not intra-arterial, seem to be safe through the intraventricular injection $^{11)}$. However, owing to the lack of recorded results, the authors were unable to extract sufficient data from the included studies regarding these complications. Thus, a systematic review for the safety of fibrinolytic drugs following $\mathrm{SAH}$ should be conducted in the near future. Because the relatively small or medium size of included studies, in addition, more multicenter, larger-scale, and prospective studies would likely clearly establish the relationship between LAFDs and the aforementioned outcomes.

\section{CONCLUSION}

The present meta-analysis and systematic review with eight RCTs identified that administration of LAFDs seemed to be effective in reducing the incidence rates of vasospasm and hydrocephalus. Thus, LAFDs could consequently reduce the mortality rates and improve the clinical outcome after SAH.

\section{CONFLICTS OF INTEREST}

No potential conflict of interest relevant to this article was reported.

\section{INFORMED CONSENT}

This type of study does not require informed consent. 


\section{AUTHOR CONTRIBUTIONS}

\author{
Conceptualization : KMJ, HHC, YSP \\ Data curation : KMJ, TKN \\ Formal analysis : KMJ, HHC, DH \\ Funding acquisition: $\mathrm{HHC}$ \\ Methodology : KMJ, HHC, DH \\ Project administration : HHC, JTK \\ Visualization : HHC \\ Writing - original draft : KMJ \\ Writing - review \& editing : HHC, TKN, JSB
}

\section{ORCID}

Kyoung Min Jang https://orcid.org/0000-0002-0452-4595

Hyun Ho Choi https://orcid.org/0000-0003-1170-6829

Taek Kyun Nam https://orcid.org/0000-0003-3151-631X

Yong Sook Park https://orcid.org/0000-0003-1152-6844

Jeong Taik Kwon https://orcid.org/0000-0002-7889-7634

Jun Soo Byun https://orcid.org/0000-0003-3210-9505

Doyeon Hwang https://orcid.org/0000-0002-0215-5319

\section{- Supplementary materials}

The online-only data supplement is available with this article at https://doi.org/10.3340/jkns.2020.0154.

\section{References}

1. Amin-Hanjani S, Ogilvy CS, Barker FG : Does intracisternal thrombolysis prevent vasospasm after aneurysmal subarachnoid hemorrhage? A meta-analysis. Neurosurgery 54 : 326-335, 2004

2. Ayling OG, Ibrahim GM, Alotaibi NM, Gooderham PA, Macdonald RL : Dissociation of early and delayed cerebral infarction after aneurysmal subarachnoid hemorrhage. Stroke 47 : 2945-2951, 2016

3. Connolly ES Jr, Rabinstein AA, Carhuapoma JR, Derdeyn CP, Dion J, Higashida RT, et al. : Guidelines for the management of aneurysmal subarachnoid hemorrhage: a guideline for healthcare professionals from the American Heart Association/American Stroke Association. Stroke 43 : 1711-1737, 2012

4. Dorsch NW : Cerebral arterial spasm--a clinical review. Br J Neurosurg 9 : 403-412, 1995

5. Drake CG : Report of World Federation of Neurological Surgeons Committee on a universal subarachnoid hemorrhage grading scale. J Neu- rosurg $68: 985-986,1988$

6. Eicker SO, Beseoglu K, Etminan N, Perrin J, Taskin A, Steiger HJ, et al. : The effect of intraventricular thrombolysis in combination with low-frequency head motion after severe subarachnoid hemorrhage: interim analysis of safety, clot clearance rate and delayed cerebral ischemia. Acta Neurochir Suppl $114: 323-328,2012$

7. Etminan N, Beseoglu K, Eicker SO, Turowski B, Steiger HJ, Hänggi D : Prospective, randomized, open-label phase II trial on concomitant intraventricular fibrinolysis and low-frequency rotation after severe subarachnoid hemorrhage. Stroke 44 : 2162-2168, 2013

8. Findlay JM, Kassell NF, Weir BK, Haley EC Jr, Kongable G, Germanson T, et al. : A randomized trial of intraoperative, intracisternal tissue plasminogen activator for the prevention of vasospasm. Neurosurgery 37 : 168-176; discussion 177-178, 1995

9. Findlay JM, Weir BK, Steinke D, Tanabe T, Gordon P, Grace M : Effect of intrathecal thrombolytic therapy on subarachnoid clot and chronic vasospasm in a primate model of SAH. J Neurosurg 69 : 723-735, 1988

10. Gerner ST, Kuramatsu JB, Abel H, Kloska SP, Lücking H, Eyüpoglu IY, et al. : Intraventricular fibrinolysis has no effects on shunt dependency and functional outcome in endovascular-treated aneurysmal SAH. Neurocrit Care $21:$ 435-443, 2014

11. Gilard V, Metayer T, Gakuba C, Langlois O, Proust F, Emery E, et al. : Intraventricular hemorrhage related to AVM rupture: description, outcomes and impact of intraventricular fibrinolysis. Clin Neurol Neurosurg 164 : 92-96, 2018

12. Giraldo EA, Mandrekar JN, Rubin MN, Dupont SA, Zhang Y, Lanzino G, et al. : Timing of clinical grade assessment and poor outcome in patients with aneurysmal subarachnoid hemorrhage. J Neurosurg 117 : 15-19, 2012

13. Hänggi $D$, Liersch J, Turowski $B$, Yong $M$, Steiger $H J$ : The effect of lumboventricular lavage and simultaneous low-frequency head-motion therapy after severe subarachnoid hemorrhage: results of a single center prospective phase II trial. J Neurosurg 108 : 1192-1199, 2008

14. Hamada J, Kai Y, Morioka M, Yano S, Mizuno T, Hirano T, et al. : Effect on cerebral vasospasm of coil embolization followed by microcatheter intrathecal urokinase infusion into the cisterna magna: a prospective randomized study. Stroke 34 : 2549-2554, 2003

15. Karamanakos PN, von und zu Fraunberg M, Bendel S, Huttunen T, Kurki $\mathrm{M}$, Hernesniemi J, et al. : Risk factors for three phases of 12-month mortality in 1657 patients from a defined population after acute aneurysmal subarachnoid hemorrhage. World Neurosurg 78 : 631-639, 2012

16. Kawamoto S, Tsutsumi K, Yoshikawa G, Shinozaki MH, Yako K, Nagata $K$, et al. : Effectiveness of the head-shaking method combined with cisternal irrigation with urokinase in preventing cerebral vasospasm after subarachnoid hemorrhage. J Neurosurg 100 : 236-243, 2004

17. Kramer AH, Roberts DJ, Holodinsky J, Todd S, Hill MD, Zygun DA, et al. : Intraventricular tissue plasminogen activator in subarachnoid hemorrhage patients: a prospective, randomized, placebo-controlled pilot trial. Neurocrit Care $21: 275-284,2014$

18. Li YH, Guo K, Zi XH, Song Z : Combining exchange of cerebrospinal fluid with small dose of urokinase injection for subarachnoid hemorrhage. J 
Cent South Univ (Med Sci) 30 : 217-220, 2005

19. Litrico S, Almairac F, Gaberel T, Ramakrishna R, Fontaine D, Sedat J, et al. : Intraventricular fibrinolysis for severe aneurysmal intraventricular hemorrhage: a randomized controlled trial and meta-analysis. Neurosurg Rev 36 : 523-530; discussion 530-531, 2013

20. Macdonald RL : Delayed neurological deterioration after subarachnoid haemorrhage. Nat Rev Neurol 10 : 44-58, 2014

21. Macdonald RL, Rosengart A, Huo D, Karrison T : Factors associated with the development of vasospasm after planned surgical treatment of aneurysmal subarachnoid hemorrhage. J Neurosurg 99 : 644-652, 2003

22. Mandava P, Martini SR, Munoz M, Dalmeida W, Sarma AK, Anderson $J A$, et al. : Hyperglycemia worsens outcome after rt-PA primarily in the large-vessel occlusive stroke subtype. Transl Stroke Res 5 : 519-525, 2014

23. Medina MG, Ledesma MD, Domínguez JE, Medina M, Zafra D, Alameda $F$, et al. : Tissue plasminogen activator mediates amyloid-induced neurotoxicity via Erk1/2 activation. EMBO J 24 : 1706-1716, 2005

24. Ramakrishna R, Sekhar LN, Ramanathan D, Temkin N, Hallam D, Ghodke $B V$, et al. : Intraventricular tissue plasminogen activator for the prevention of vasospasm and hydrocephalus after aneurysmal subarachnoid hemorrhage. Neurosurgery 67 : 110-117; discussion 117, 2010

25. Reilly C, Amidei C, Tolentino J, Jahromi BS, Macdonald RL : Clot volume and clearance rate as independent predictors of vasospasm after aneurysmal subarachnoid hemorrhage. J Neurosurg 101 : 255-261, 2004

26. Shi L, Xu L, Shi L, Brandon D, Chen S, Zhang J : Intraventricular recombi- nant tissue plasminogen activator in treatment of aneurysmal intraventricular hemorrhage: a meta-analysis. Curr Drug Targets 18 : 13991407, 2017

27. Staykov D, Kuramatsu JB, Bardutzky J, Volbers B, Gerner ST, Kloska SP, et al. : Efficacy and safety of combined intraventricular fibrinolysis with lumbar drainage for prevention of permanent shunt dependency after intracerebral hemorrhage with severe ventricular involvement: a randomized trial and individual patient data meta-analysis. Ann Neurol 81 : 93-103, 2017

28. Varelas PN, Rickert KL, Cusick J, Hacein-Bey L, Sinson G, Torbey M, et al. : Intraventricular hemorrhage after aneurysmal subarachnoid hemorrhage: pilot study of treatment with intraventricular tissue plasminogen activator. Neurosurgery 56 : 205-213; discussion 205-213, 2005

29. Wang D, Liu J, Norton C, Liu M, Selim M : Local fibrinolytic therapy for intraventricular hemorrhage: a meta-analysis of randomized controlled trials. World Neurosurg 107 : 1016-1024.e1, 2017

30. Wilson TJ, Stetler WR Jr, Davis MC, Giles DA, Khan A, Chaudhary N, et al. : Intraventricular hemorrhage is associated with early hydrocephalus, symptomatic vasospasm, and poor outcome in aneurysmal subarachnoid hemorrhage. J Neurol Surg A Cent Eur Neurosurg 76 : 126-132, 2015

31. Yamamoto T, Esaki T, Nakao Y, Mori K : Efficacy of low-dose tissueplasminogen activator intracisternal administration for the prevention of cerebral vasospasm after subarachnoid hemorrhage. World Neurosurg $73: 675-682,2010$ 